\title{
Pblebotomus (Larroussius) mariae n. sp. (Diptera-Psychodidae)
}

\author{
par J.-A. RIOUX, H. CROSET, N. LEGER et H. BAILLY-CHOUMARA \\ Laboratoire d'Ecologie médicale et de Pathologie parasitaire ( $\mathrm{P}^{\mathrm{r}} \mathrm{J} .-\mathrm{A}$. Rioux) \\ Faculté de Médecine, rue Auguste-Broussonnet, F 34000 Montpellier \\ Laboratoire de Parasitologie, Faculté de Pharmacie, \\ 4, avenue de l'Observatoire, $F 75006$ Paris \\ et Institut scientifique chérifien, Rabat, Maroc
}

\section{Résumé.}

Les auteurs décrivent une nouvelle espèce de Phlébotome, Phlebotomus mariae n. sp., rattachée au sous-genre Larroussius. Celle-ci se distingue par la forme du pénis à bords subparallèles, long, grêle, symétrique, et très faiblement évasé à l'extrémité apicale.

\section{Summary.}

Phlebotomus (Larroussius) mariae n. sp. (Diptera, Psychodidae).

The authors describe a new Sandfly linked with the Larroussius sub-genus, Phlebotomus mariae n. sp. (Diptera, Phlebotominae). This one is particularized by the shape of its penis wich is long, slender, symmetric and faintly flared at its apical end so that its sides are subparallel.

Dans le cadre d'une première enquête sur les Phlébotomes du Sud marocain (1), nous avons pu récolter un abondant matériel, le long d'un itinéraire-transect de $825 \mathrm{~km}$, depuis Meknès jusqu'à Ouarzazate, via Taouz. Sur un lot de 9.115 mâles capturés par la technique des pièges adhésifs, 6 exemplaires, morphologiquement identiques, n'ont

(1) R.C.P. $\mathrm{n}^{\circ} 249$ (C.N.R.S.) : Etude de certains milieux du Maroc et de leur évolution récente. 
pu être rapportés à l'une des espèces de Larroussius actuellement connue. Nous en proposons la description sous le binôme de Phlebotomus mariae (2).

\section{Deseription (3).}

\section{A. - Male.}

1) Taille (coxite excepté) :

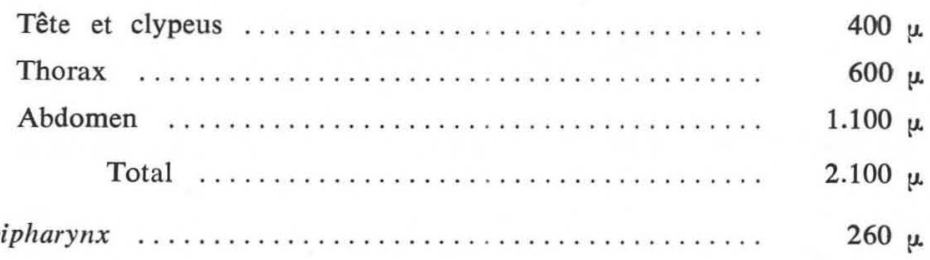

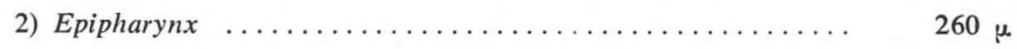

3) Palpes: longueur totale: $970 \mu$; dimensions des articles se décomposant comme suit :

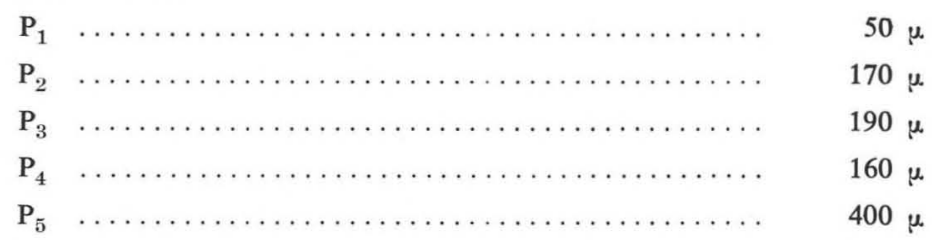

Formule palpale: 1, 4, 2, 3, 5 .

Rapport P/E : 3,35.

4) Ailes (fig. $1 \mathrm{a}$ ):

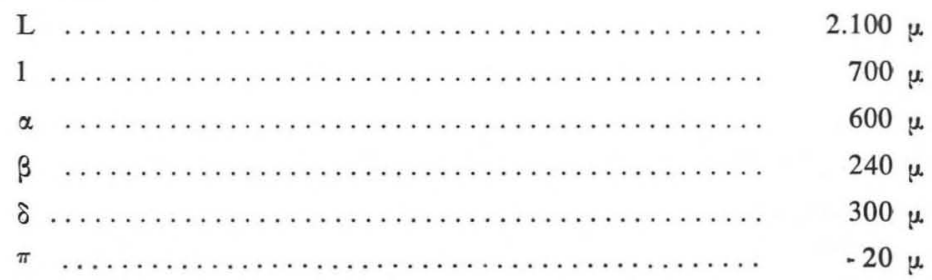

5) Armature génitale (fig. 1c, 2, 3, 4 et 5):

Coxite $340 \mu$

Style $190 \mu$

Valve pénienne (pénis) $150 \mu$

Paramère $230 \mu$

(2) Dédiée à $\mathbf{M}^{\text {1le }}$ Marie Maistre, technicienne du C.N.R.S.

(3) Le mauvais état des échantillons, dû au traumatisme du piège adhésif, nous a obligés à réaliser les mensurations à partir de trois exemplaires mâles. 
Lobe latéral $380 \mu$

Papille anale $160 \mu$

Pompe génitale $140 \mu$

Soie du coxite

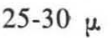

Rapport Pénis/Coxite : 0,44.

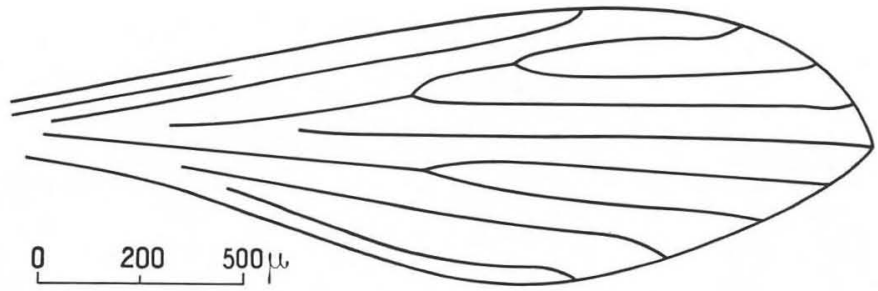

$a$
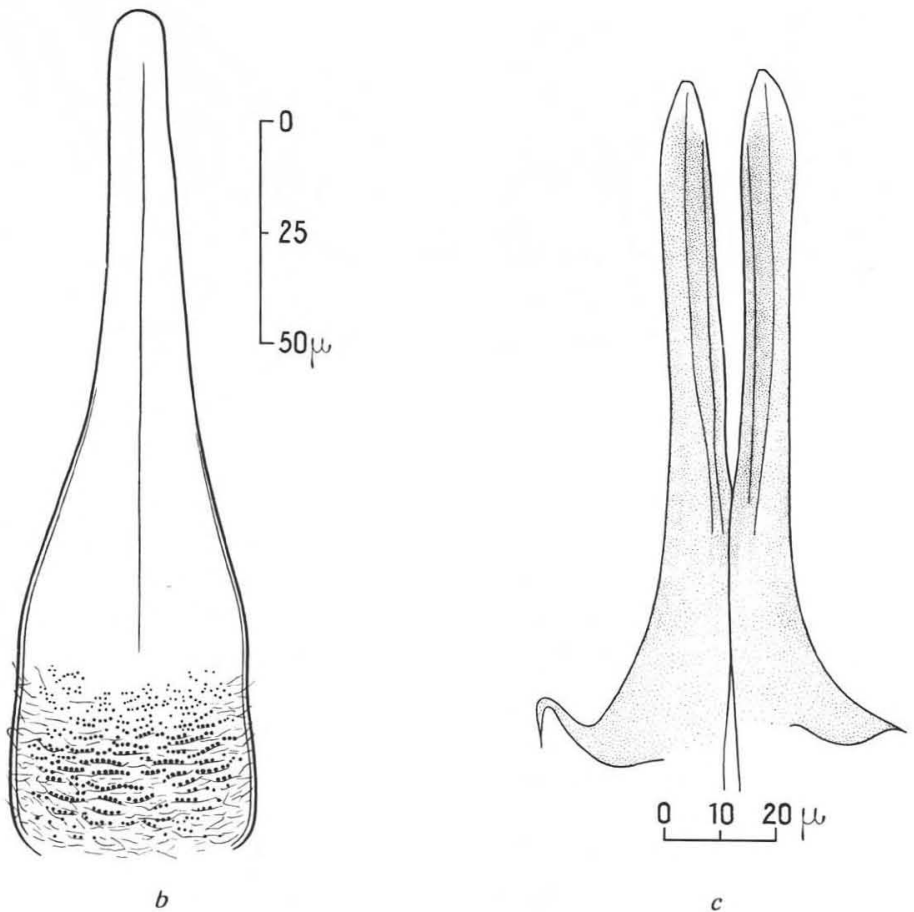

FIG. 1. - Phlebotomus mariae n. sp. đ․ $a$ : Aile, $b$ : Pharynx, $c$ : Valves péniennes 
Les caractères biométriques énumérés ci-dessus (4) ne suffisent pas, à eux seuls, pour séparer Phlebotomus mariae des espèces affines du sous-genre Larroussius. Il en est de même de l'armature pharyngienne (fig. 1, b), dont on connaît d'ailleurs la faible différenciation chez les Phlébotomes mâles. En fait, seule la structure des genitalia, et singulièrement celle des valves péniennes, permet d'individualiser aisément Phlebotomus mariae.

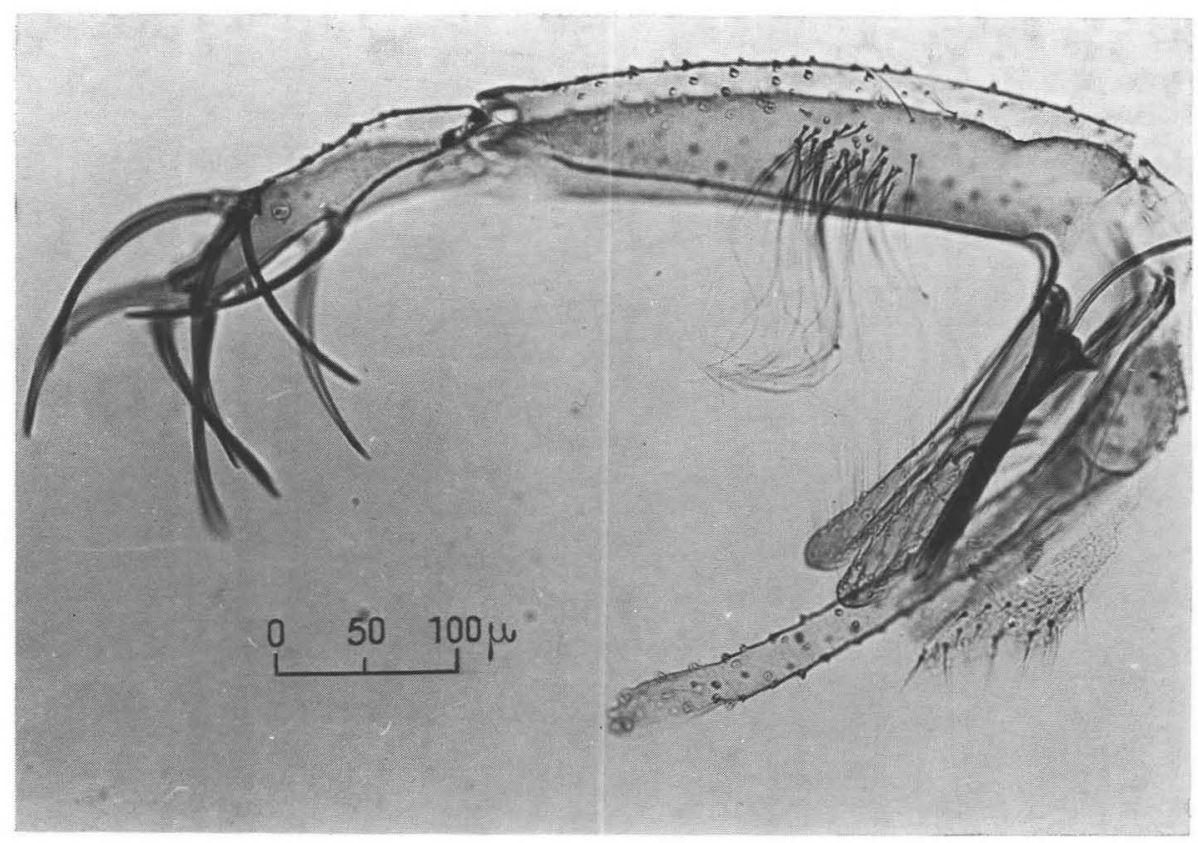

FIG. 2. - Phlebotomus mariae n. sp. Genitalia ฮै.

Les coxites sont porteurs d'une touffe interne de 25 à 30 soies, groupées en une tache relativement nette. Les styles sont pourvus de 5 épines (5) bien développées. Les lobes latéraux sont grêles et plus longs que le coxite. Quant aux valves péniennes, sur lesquelles repose la diagnose spécifique, elles sont longues, étroites, à bords subparallèles et très discrètement renflées à leur extrémité libre. Les filaments péniens débouchent exactement en leur sommet.

Un tel aspect sépare nettement Phlebotomus mariae de Phlebotomus major Annandale, 1910 (valves plus grêles, terminées en «baguette de tambour»; orifice du

(4) La biométrie antennaire (longueurs des articles, épines géniculées, formule antennaire) aurait peut-être apporté un ou plusieurs éléments de discrimination. Malheureusement, le traumatisme infligé aux échantillons par les pièges adhésifs ne permet pas actuellement de faire état de ces caractères.

(5) L'un des exemplaires récoltés ne comportait que quatre épines sur le style (fig. 4), anomalie fréquente chez les Phlébotomes (E. Abonnenc, S. Poinsot et J.-A. Rioux, 1971). 


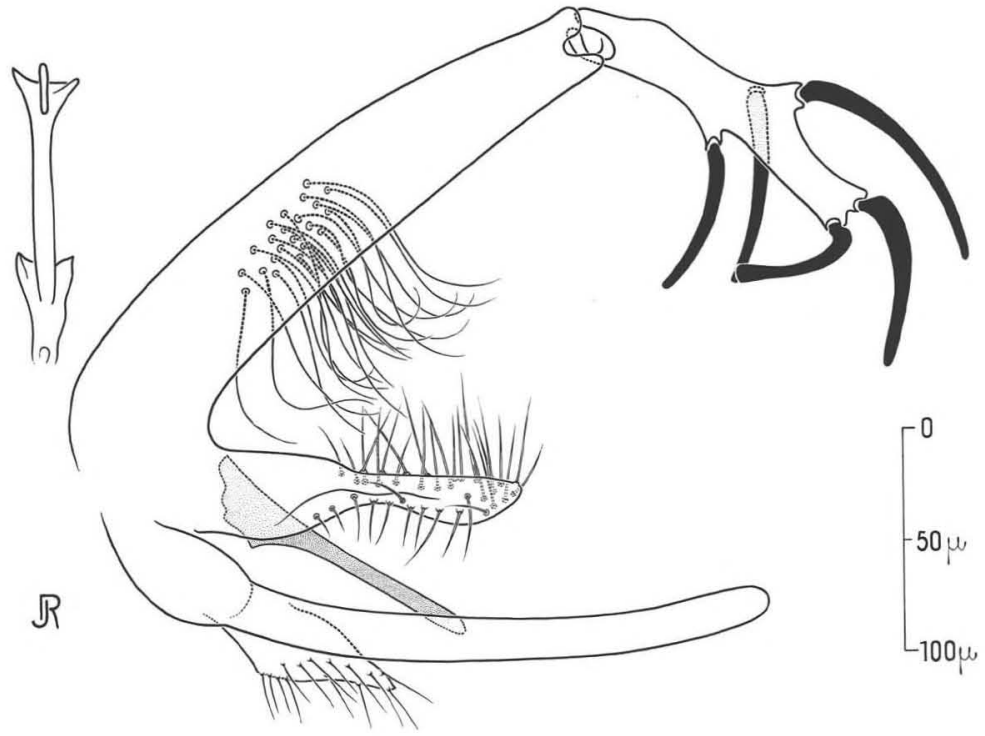

FIG. 3. - Phlebotomus mariae n. sp. Genitalia §ึ.

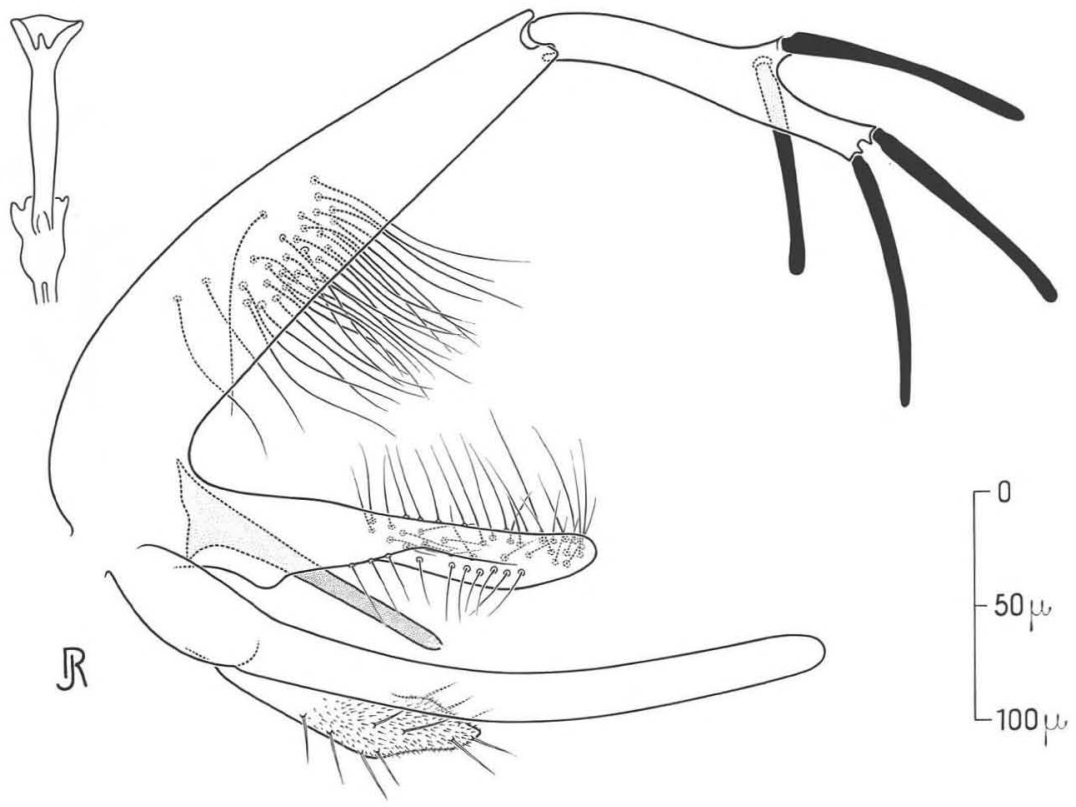

FIG. 4. - Phlebotomus mariae n. sp. Genitalia ở. Style tératologique à quatre épines. 


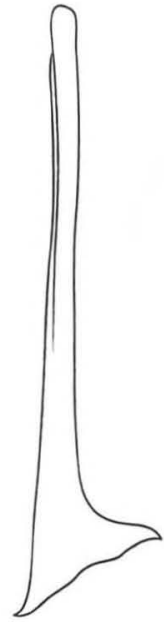

major

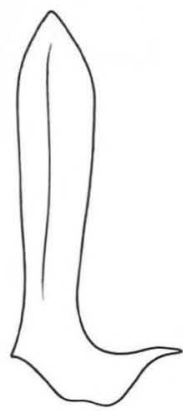

chadlii

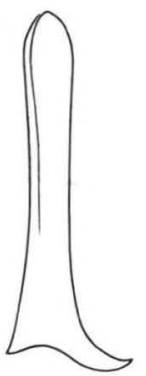

ariasi
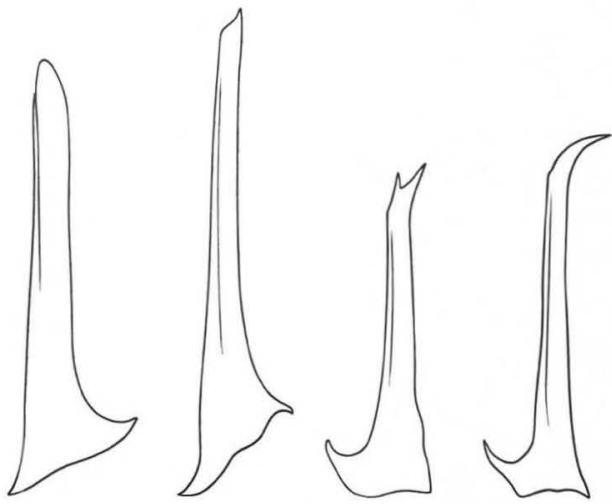

mariae langeroni perniciosus longicuspis

FIG. 5. - Valves péniennes.

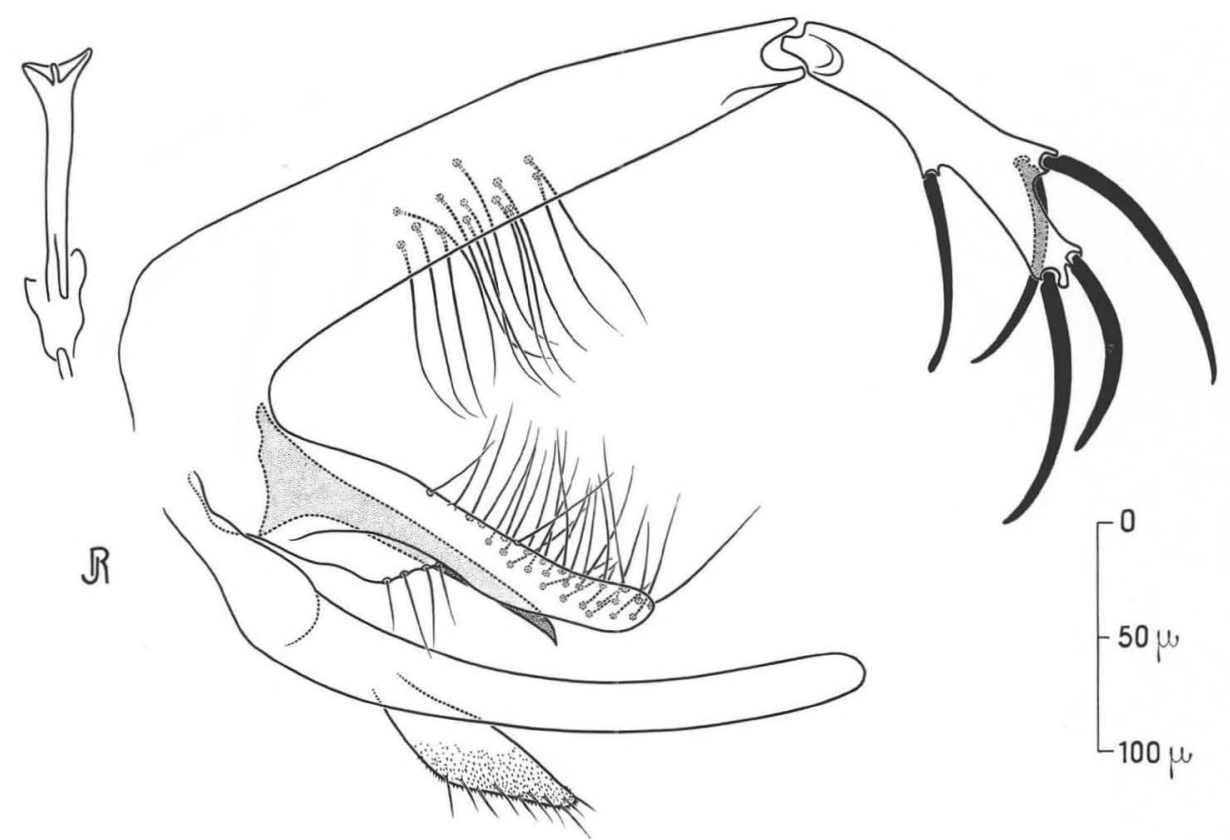

FIG. 6. - Phlebotomus langeroni Nitzulescu, 1930. Genitalia $\delta$ (exemplaire récolté aux environs de Tunis). 
filament sublatéral, fig. 5), Phlebotomus langeroni Nitzulescu, 1930 (valves terminées par une épine courte et déjetée, fig. 5 et 6), Phlebotomus longicuspis Nitzulescu, 1930 (valves terminées en une pointe longue et incurvée, fig. 5) et Phlebotomus perniciosus Newstead, 1911 (valves bicuspides, fig. 5). Quant à Phlebotomus ariasi Tonnoir, 1921 et Phlebotomus chadlii Rioux, Juminer et Gibily, 1966, leurs valves sont beaucoup plus trapues et nettement renflées à l'apex, soit sous la forme d'un ovale régulier pour le premier (aspect en «battant de cloche», fig. 5 et 7), soit d'un évasement sublosangique pour le second (aspect en glaive, fig. 5 et 8 ). D'ailleurs, la présence dans un même biotope (TM 94 et TM 96) de Phlebotomus ariasi, Phlebotomus chadlii et Phlebotomus mariae est un argument supplémentaire en faveur de l'autonomie de ces trois espèces (cf. ci-après).

\section{B. - Femelle.}

Pour l'instant, la femelle de Phlebotomus mariae reste inconnue. Certains exemplaires $q$ provenant des mêmes stations et pourvus d'un pharynx fortement ponctué appartiennent peut-être à cette espèce.

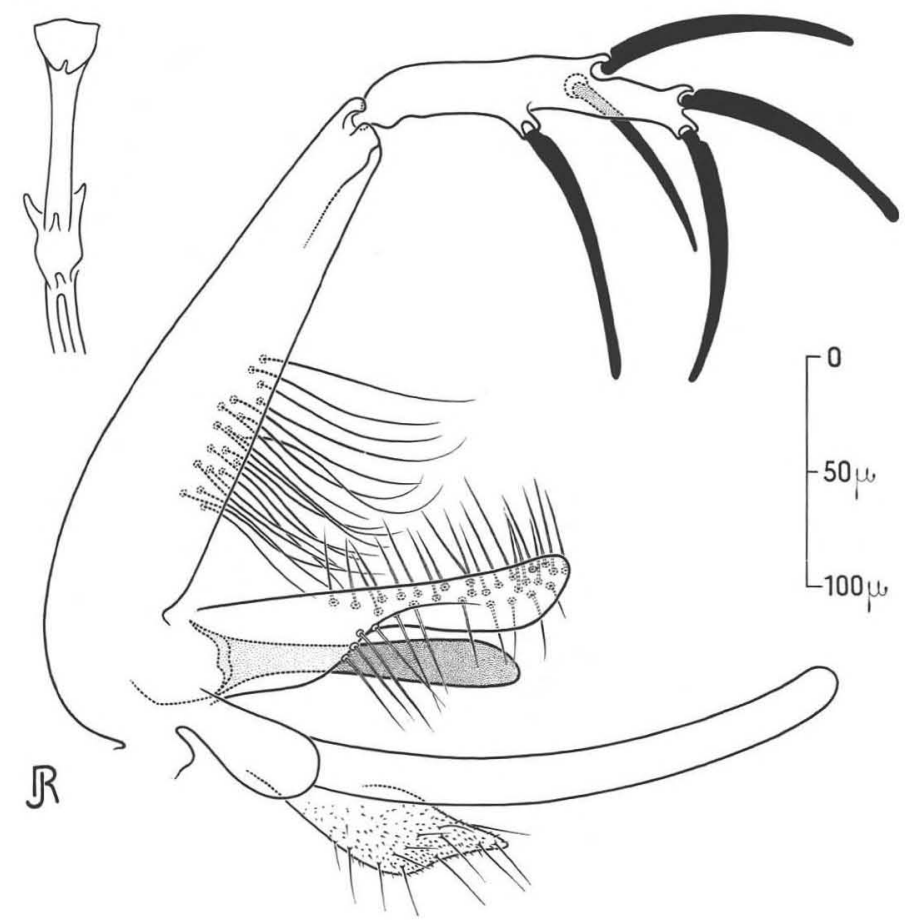

FIg. 7. - Phlebotomus ariasi Tonnoir, 1921. Genitalia $\sigma^{7}$ (exemplaire récolté aux environs de Tunis; locus classicus) 


\section{Localités. Espèces associées.}

Les stations de Phlebotomus mariae sont toutes situées sur le versant sud du HautAtlas, aux environs de Tinerhir et de Boumalne-du-Dadès.

$1^{\circ}$ Gorges DU Todhra (TM 87), à proximité des «Buvettes », à $15 \mathrm{~km}$ au nord de Tinerhir. Altitude $1.450 \mathrm{~m}$. Etage aride-froid. Hautes falaises exposées au nord. (Atlantoxerus getulus et Eliomys quercinus). 140 pièges placés le 24-9-72 et retirés le 26-9-72.

Phlebotomus mariae, 1 mâle en compagnie de :

Phlebotomus (Phlebotomus) papatasi (Scopoli, 1786) .......... 1 \% 1 o

Phlebotomus (Paraphlebotomus) sergenti Parrot, $1917 \ldots \ldots \ldots \ldots . \quad 1 \% 40.6$

Phlebotomus (Paraphlebotomus) chabaudi Croset, Abonnenc et

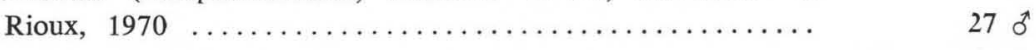

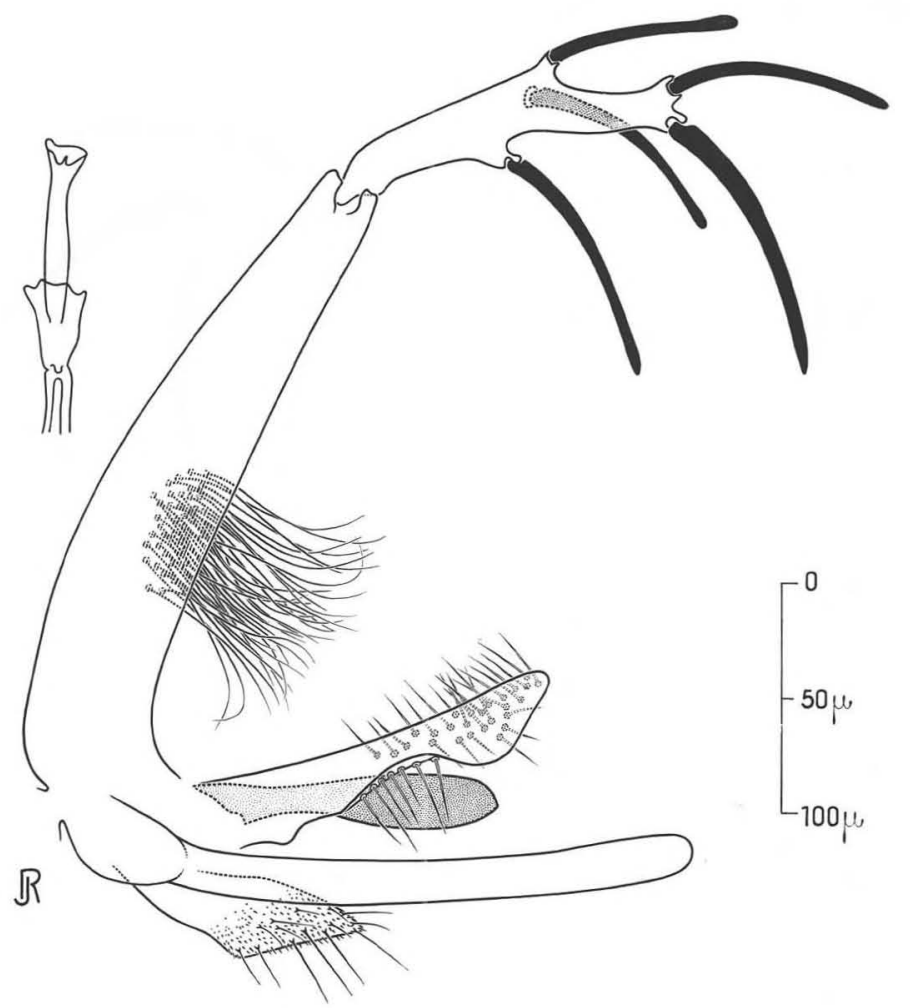

FIG. 8. - Phlebotomus chadlii Rioux, Juminer et Gibily, 1966. Genitalia ฮै (exemplaire récolté dans les Gorges du Dadès; station TM 94). 
Phlebotomus (Paraphlebotomus) alexandri Sinton, $1928 \ldots \ldots \ldots$

Phlebotomus (Larroussius) longicuspis Nitzulescu, 1930 .......

Sergentcmyia (Sergentomyia) minuta parroti (Adler et Theodor,

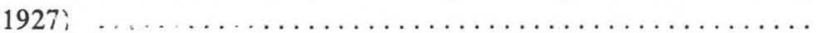

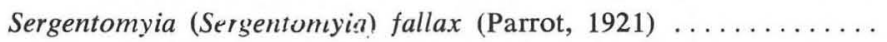

Sergentomyia (Sergentomyia) antennata (Newst., 1912) .......

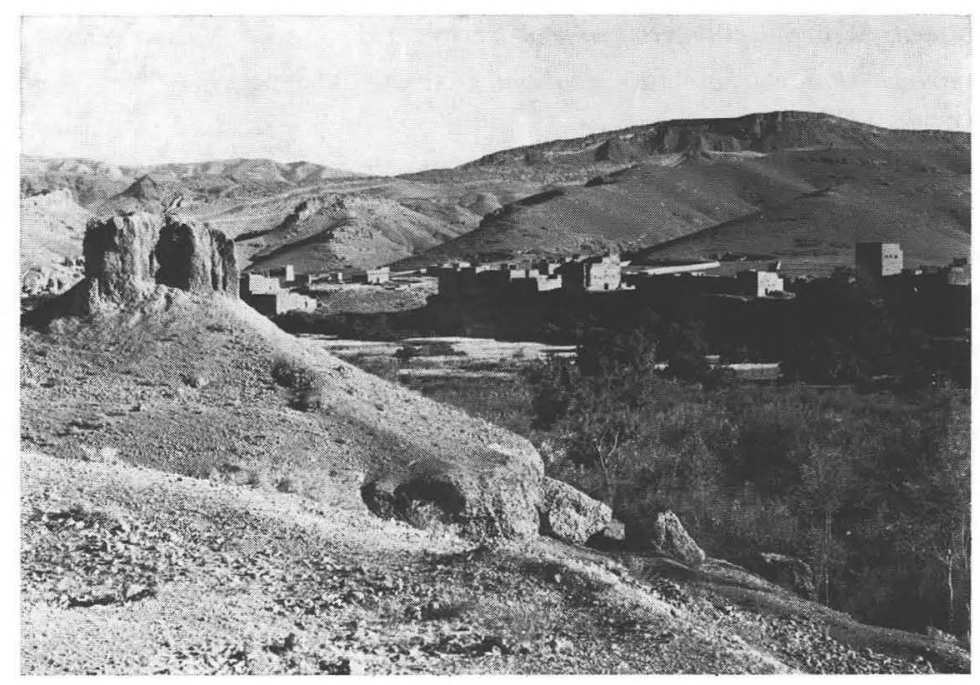

FIG. 9. - Vallée du Dadès au nord de Boumalne (station TM 96), station de Phlebotomus mariae n. sp.

$2^{\circ}$ Gorges Du Dadès (TM 94), à $12 \mathrm{~km}$ au nord de Boumalne (fig. 9). Altitude $1.640 \mathrm{~m}$. Etage aride-froid. Rochers, encorbellements et grottes à la sortie de village. Exposition est. 86 pièges placés le 26-9-72 et retirés le 29-9-72.

Phlebotomus mariae, 3 mâles en compagnie de:

Phlebotomus (Phlebotomus) papatasi (Scopoli, 1786) ......... 1 우

Phlebotomus (Paraphlebotomus) sergenti Parrot, $1917 \ldots \ldots \ldots \ldots$

Phlebotomus (Paraphlebotomus) chabaudi Croset, Abonnenc et

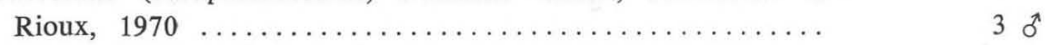

Phlebotomus (Paraphlebotomus) alexandri Sinton, $1928 \ldots \ldots \ldots .20 . \ldots$

Phlebotomus (Larroussius) ariasi Tonnoir, $1921 \ldots \ldots \ldots \ldots \ldots \ldots / 20 . \ldots 20$

Phlebotomus (Larroussius) chadlii Rioux, Juminer et Gibily, 1966$\} \begin{array}{ll}2 & 9 \\ 5 & \delta^{\star}\end{array}$

Phlebotomus (Larroussius) longicuspis Nitzulescu, $1930 \ldots \ldots \ldots . \quad 8 \% 46 \delta^{\pi}$ 
Sergentomyia (Sergentomyia) minuta parroti (Adler et Theodor, 1927) $16 \subsetneq 17 \delta^{7}$

Sergentomyia (Sergentomyia) fallax (Parrot, 1921)

$3^{\circ}$ Gorges du Dadès (TM 96), à $5 \mathrm{~km}$ au nord de Boumalne. Altitude $1.610 \mathrm{~m}$. Etage aride-froid. Encorbellements sous grès et calcaires. Exposition est. 49 pièges placés le 26-9-72 et relevés le 29-9-72.

Phlebotomus mariae, 2 mâles en compagnie de:

Phlebotomus (Phlebotomus) papatasi (Scopoli, 1786) ...........

Phlebotomus (Paraphlebotomus) sergenti Parrot, $1917 \ldots \ldots \ldots$.

Phlebotomus (Paraphlebotomus) chabaudi Croset, Abonnenc et

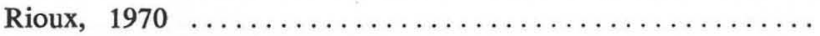

Phlebotomus (Paraphlebotomus) alexandri Sinton, $1928 \ldots \ldots \ldots \ldots$

Phlebotomus (Larroussius) ariasi Tonnoir, $1921 \ldots \ldots \ldots \ldots \ldots$.

Phlebotomus (Larroussius) chadlii Rioux, Juminer et Gibily, 1966

Phlebotomus (Larroussius) longicuspis Nitzulescu, 1930 .......

Sergentomyia (Sergentomyia) minuta parroti (Adler et Theodor,

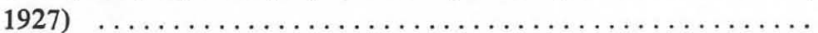

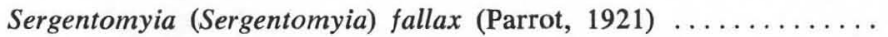

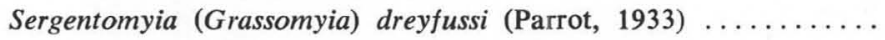

Sergentomyia (Sintonius) christophersi (Sinton, 1927) ..........

Type et cotype déposés dans les collections du Laboratoire d'Ecologie médicale, Faculté de Médecine de Montpellier.

\section{Bibliographie}

Abonnenc (E.), 1972. - Les Phlébotomes de la région éthiopienne (Diptera, Psychodidae). Cah. O.R.S.T.O.M., 55, 289 p.

—, Poinsot (S.) et Rioux (J.-A.), 1971. - Tératologie des Phlébotomes (Diptera, Psychodidae). Révision et nouvelles observations. Cah. O.R.S.T.O.M., sér. Ent. Méd., 9, 307-326.

Annandale (N.), 1910. - The indian species of Papataci fly (Phlebotomus). Rec. Ind. Mus., 4, 35-52.

Bailly-Choumara (H.), Abonnenc (E.) et Pastre (J.), 1971. - Contribution à l'étude des Phlébotomes du Maroc (Diptera, Psychodidae). Données faunistiques et écologiques. Cah. O.R.S.T.O.M., 9, 431-460.

Croset (H.), 1969. - Ecologie et systématique des Phlebotomini (Diptera, Psychodidae) dans deux foyers, français et tunisien, de Leishmaniose viscérale. Essai d'interprétation épidémiologique. Thèse Sc., Montpellier, 516 p. (ronéo).

Newstead (R.), 1911. - The Papataci flies (Phlebotomus) of the Maltese Islands. Ann. Trop. Med. Parasit., 5, 139-187. 
Nitzulescu (V.), 1930. - Sur une variété de Phlebotomus perniciosus. Ann. Parasit. hum. comp., 8, 382-385.

-, 1930. - Phlebotomus langeroni n. sp. et P. langeroni var. longicuspis n. var. de DouarShott (Tunisie). Ann. Parasit. hum. comp., 8, 547-553.

Rioux (J.-A.), Juminer (B.) et Gibily (H.), 1966. - Phlebotomus (Phlebotomus) chadlii n. sp. (Diptera-Psychodidae). Ann. Parasit. hum. comp., 41, 83-89.

Tonnolr (A.), 1921. - Une nouvelle espèce européenne du genre Phlebotomus (Phlebotomus ariasi). Ann. Soc. Ent. de Belgique, 61, 53-56.

\section{ERRATUM}

Nématodes Trichostrongyles du genre Molineus Cameron 1923, parasites d'Insectivores malgaches, par M.-Cl. Durette-Desset.

Dans cet article publié dans le $\mathrm{n}^{\circ} 5$, septembre-octobre 1973, pp. 677-698, il faut lire: Molineus albignaci a été trouvé chez Leptogale gracilis et non chez Nesogale dobsoni comme il a été indiqué par erreur dans les résumés français et anglais. 